\title{
Desempenho-Complexidade de Sistemas DS-UWB em Canais Multipercursos Densos
}

\author{
Lisiane C. Heringer ${ }^{1}$, Fernando Ciriaco $^{2}$, Bruno A. Angélico ${ }^{3}$, Taufik Abrão ${ }^{4}$ \& Paul Jean E. Jeszensky ${ }^{5}$
}

\begin{abstract}
Resumo-A tecnologia de comunicação de banda ultra larga (UWB - Ultra Wideband) possui propriedades que a tornam uma candidata promissora na implementação de comunicações de curto alcance em ambientes de multipercursos densos e sujeitos à interferência de banda estreita. Dentre as versões UWB impulsivas atualmente investigadas, o sistema UWB de seqüência direta (DS-UWB) tem sido considerado como um dos esquemas mais atrativos para a quarta geração das comunicações sem fio (4G). Neste trabalho são combinados os conceitos de diversidade temporal, espacial e recepção multiusuário, tendo em vista a melhoria de desempenho através do uso do receptor Rake, do cancelador de interferência paralelo (PIC - Parallel Interference Cancellation) e de múltiplas antenas receptoras. A combinação destas estratégias permite reduzir o efeito da interferência de múltiplo acesso (MAI - Multiple Access Interference), aumentando a capacidade desses sistemas. Análises do compromisso desempenho versus complexidade indicaram que é preferível aumentar o número de antenas e/ou de ramos do receptor Rake ao invés de incrementar o número de estágios PIC.
\end{abstract}

Palavras-Chave-Comunicação de banda ultra larga, DSUWB, desempenho-complexidade, detecção multiusuário.

Abstract-Ultra wideband (UWB) has properties that make it a viable candidate for short-range communications in dense multipath environments. Direct-Sequence UWB (DS-UWB) is considered one of the most attractive and promising system for the future fourth generation (4G) wireless communications scenario. Among other possible schemes the impulsive UWB version are investigated in this paper. The concept of space diversity, with the application of multiple receiver antennas, was explored for the performance improvement, and multiuser detection, based on parallel interference cancellation (PIC Parallel Interference Cancellation), was used in order to suppress the effects of the MAI and to increase capacity and performance. The application of both techniques was analyzed considering its capability to reach the performance-complexity improvement promises.

Keywords-Ultra wide band system, DS-UWB, performancecomplexity. multiuser detection.

\section{INTRODUÇÃO}

Nos últimos anos, a comunicação de banda ultra larga (UWB) tem despertado interesse de pesquisadores bem como de empresas do ramo de telecomunicações, devido à promessa de fornecimento de altas taxas de dados combinadas ao

\footnotetext{
${ }^{1}$ Estudante de mestrado do Depto Eng. Elétrica da UEL. E-mail: lisianech@yahoo.com.br

${ }^{2,3}$ Estudante de doutorado do PTC - Depto de Engenharia de Telecomunicações e Controle da EPUSP. E-mail: fciriaco@1cs.poli.usp.br e angelico@1cs.poli.usp.br

${ }^{4}$ Professor Adjunto do DEEL - Departamento Eng. Elétrica da UEL - PR, Brazil. E-mail: taufik@uel.br.

${ }^{5}$ Professor Titular do PTC - Departamento de Engenharia de Telecomunicações e Controle, EPUSP. E-mail: pjj@lcs.poli.usp.br
}

baixo custo e ao reduzido consumo de potência. Os sinais de rádio de UWB empregam a transmissão de pulsos muito curtos cujo espectro se estende através de uma larga faixa de freqüências [1]. Esses pulsos de período extremamente curtos têm normalmente duração de alguns nanosegundos ou mesmo centenas de picosegundos, resultando em uma elevada taxa de transmissão [1]-[4]. Face a suas características de alta taxa de transmissão e baixo consumo de potência, as comunicações de UWB são indicadas para sistemas de comunicação de pequeno alcance, especialmente em redes pessoais sem fio (WPAN Wireless Personal Area Networks).

Teoricamente, por utilizar níveis de potência muito baixos, sistemas de comunicação UWB podem coexistir com outras tecnologias na mesma faixa de freqüências de operação, tais como as tecnologias já existentes Wi-Fi (padrões IEEE 802.11x), GSM e Bluetooth.

O UWB foi inicialmente desenvolvido para aplicações militares; em fevereiro de 2002 a agência reguladora norteamericana FCC (Federal Communications Commission) expediu uma regulamentação para o uso comercial dessa tecnologia, estabelecendo os limites para a banda e potência de transmissão. Porém, os esforços para sua padronização continuam. Os sinais UWB devem possuir largura de banda fracionária ${ }^{1}$ maior do que $20 \%$ ou ocupar pelo menos 500 $\mathrm{MHz}$ de banda.

A tecnologia de rádio UWB impulsiva pode ser combinada com técnicas de espalhamento espectral tradicionais, para aumentar a capacidade de múltiplo acesso. Grande parte das pesquisas em sistemas UWB de múltiplo acesso têm como base o espalhamento espectral por saltos no tempo (TH Time Hopping), usando sinais modulados por posição de pulso (PPM - Pulse Position Modulation) [4]. Espalhamento espectral por seqüência direta (DS-SS - Direct Sequence Spread Spectrum) também é uma técnica de múltiplo acesso propícia e bem conhecida para aplicações em UWB. No sistema DSUWB, múltiplos pulsos são transmitidos por período de bit usando modulação bipolar para cada pulso, baseado em um certo código de espalhamento [5]. Este método tem muitas propriedades atrativas, incluindo uma baixa relação entre a potência de pico e a potência média e robustez à MAI.

Como o pulso transmitido possui período extremamente curto, há muitas componentes multipercusos discerníveis no receptor. Portanto, o receptor RAKE pode ser usado para obter ganho de diversidade. A análise de receptores RAKE em sistemas UWB sujeitos a ambientes multipercursos densos

\footnotetext{
${ }^{1}$ Largura de banda fracionária é definida por: $B_{f}=2 \frac{f_{H}-f_{L}}{f_{H}+f_{L}}$, onde $f_{H}$ e $f_{L}$ representam as freqüências superior e inferior de $-10 \mathrm{~dB}$.
} 
é discutida em [6]-[10].

\section{Modelo do Sistema e Canal UWB}

O esquema de transmissão DS-UWB é obtido multiplicando-se o $n$-ésimo bit de informação $b^{n}$ por um código de espalhamento cujo chip é formatado a partir do emprego de pulsos extremamente estreitos. O sinal transmitido pelo $u$-ésmo usuário pode ser escrito como [11]:

$$
s_{u}(t)=\sum_{n=-\infty}^{\infty} \sqrt{E_{b}} b_{u}^{n} \sum_{k=0}^{N_{c}-1} a_{u, k} p\left(t-n T_{b}-k T_{c}\right)
$$

onde $N_{c}$ é o número de chips, $a_{u, k} \in\{-1,1\}$ é o $k$-ésimo chip da seqüência PN do $u$-ésimo usuário [12]. Neste trabalho, a forma do pulso $p(t)$ é modelada como a segunda derivada do pulso Gaussiano:

$$
p(t)=\frac{A \cdot 4 \pi}{\tau_{p}^{2}} \cdot e^{-2 \pi\left(\frac{t}{\tau_{p}}\right)^{2}} \cdot\left(1-\frac{A \cdot 4 \pi t^{2}}{\tau_{p}^{2}}\right)
$$

onde $\tau_{p}$ define o fator de formatação de pulso.

\section{A. Modelo do Canal UWB}

O modelo de canal escolhido pelo corpo de padronização IEEE 802.15.3a e utilizado neste artigo é similar ao descrito em [2], [3], que é baseado no modelo Saleh-Valenzuela (SV) [13] com algumas modificações. No modelo SV original, as estatísticas das amplitudes são descritas por uma distribuição Rayleigh. Porém, medidas realizadas em campo indicam que as amplitudes não seguem uma distribuição estatística de Rayleigh. Recomenda-se em [2], [3] a utilização de uma distribuição log-normal.

No modelo SV verifica-se o agrupamento dos raios (clustering) na chegada ao receptor. Tanto os tempos de chegada dos clusters quanto os dos raios dentro de um cluster são modelados estatística e independentemente através de um processo de Poisson ${ }^{2}$ [14]. O modelo em questão tem resposta impulsiva em tempo discreto dada por [2], [3]:

$$
h_{i}(t)=X_{i} \sum_{q=0}^{Q-1} \sum_{k=0}^{K-1} \alpha_{k, q}^{i} \delta\left(t-T_{q}^{i}-\tau_{k, q}^{i}\right)
$$

onde $i$ refere-se à $i$-ésima realização do canal; $K$ é o número de multipercursos e $Q$ o número de clusters; $\alpha_{k, q}^{i}$ é o $k$ ésimo coeficiente de ganho multipercurso, referente ao $q$ ésimo cluster e à $i$-ésima realização do canal; $T_{q}^{i}$ é o atraso do $q$-ésimo cluster; $\tau_{k, q}^{i}$ é o atraso do $k$-ésimo componente multipercurso relativo ao $q$-ésimo tempo $\left(T_{q}^{i}\right)$ de chegada do cluster; $X_{i}$ representa o efeito do sombreamento e $\delta(t)$ é a função delta de Dirac.

\footnotetext{
${ }^{2}$ Um processo de Poisson é caracterizado por variáveis aleatórias independentes com mesma distribuição exponencial, representando os intervalos de tempo entre ocorrências de certos eventos.
}

\section{B. Sinal Recebido}

Com isso, o sinal DS-UWB recebido pode ser expresso como [15]:

$$
r(t)=\sum_{u=1}^{U} s_{u}(t) * h_{u}(t)+n_{s i}(t)+n_{\text {mai }}(t)+\eta(t)
$$

onde $n_{s i}$ corresponde à parcela da auto-interferência (SI, SelfIntererence), $n_{m a i}$ corresponde à interferência de múltiplo acesso e $\eta$ ao ruído Gaussiano branco aditivo (AWGN, $A d$ ditive White Gaussian Noise), com densidade espectral de potência bilateral dada por $N_{0} / 2$. O símbolo “*” representa o operador convolução.

\section{Arranjo de Antenas e Rake Seletivo}

Por simplicidade, o sistema DS-UWB operará com uma antena transmissora e até $M$ antenas receptoras, configurando um arranjo linear $M \times 1$, com $M \leq 3$.

Cada elemento do arranjo de antenas é equipado com um Rake seletivo (SRake) contendo $L_{f}$ ramos (fingers) para o processamento das réplicas mais intensas do sinal de interesse.

Correlacionadores espaço-tempo processam os sinais recebidos que são separados no espaço e no tempo. Os ramos selecionados pelo SRake são então combinados coerentemente a partir da regra de combinação de máxima razão (MRC, Maximum Ratio Combining). O restante dos percursos não selecionados são tratados como SI. O receptor DS-UWB com diversidade espaço-temporal tratado neste trabalho é similar ao analisado em [15] e [16] e por brevidade não será reproduzido.

Assim como em [2], [17], assume-se desvanecimento independente para cada cluster e para cada raio dentro dos clusters. Adicionalmente, também foi considerado desvanecimento independente em cada elemento de antena. Note-se que uma antena adequada para sistemas UWB apresenta dimensões da ordem de $16 \times 3 \mathrm{~mm}$, indicando que não há maiores problemas com o aumento de volume/espaço necessário para acomodar um arranjo de 2 a 4 antenas [18].

\section{Detecção Multiusuário}

A detecção multiusuário (MUD) é uma estratégia desenvolvida para reduzir ou mesmo suprimir o efeito da MAI, o que aumenta a capacidade e o desempenho de sistemas de múltiplo acesso. $\mathrm{O}$ fato da detecção multiusuário ótima ser demasiadamente complexa para implementações práticas, motivou o desenvolvimento de várias estratégias sub-ótimas: o detector linear [19], o detector de decisão realimentada, ZFDF (Zero Forcing Decision Feedback), o cancelador de interferência sucessivo, SIC (Sucessive Interference Cancellation), e o cancelador de interferência paralelo, PIC, além das versões híbridas combinando-se cancelamento paralelo e sucessivo em grupos de usuários (Groupwise), G-PIC e G-SIC.

1) Canceladores de Interferência Subtrativo: Detectores MUD baseados no cancelamento de interferência (IC) subtrativo estimam os sinais dos usuários interferentes e utilizam estas estimativas para reconstruir a parcela interferente e subtraí-la (cancelá-la) do sinal do usuário de interesse, podendo envolver a reconstrução dos sinais interferentes em banda 
passante ou em banda base [20], [21]. Dentre os esquemas de cancelamento de interferência subtrativo, dois se destacam: o SIC e o PIC [22].

O detector PIC estima e subtrai, paralelamente, toda a MAI para cada usuário. O primeiro estágio é essencialmente um receptor convencional (Rake), produzindo estimativas para os sinais de todos os usuários; a única diferença é que a decisão de bit não é feita neste ponto. No segundo estágio, as estimativas melhoradas são então subtraídas do sinal recebido. Cada estágio PIC introduz atraso de um bit (período de bit, $T$ ) ao processo de detecção do sinal de interesse. Este processo pode ser repetido, gerando múltiplos estágios. O esquema de cancelamento de interferência paralelo adotado neste trabalho emprega um decisor abrupto e cancelamento total em cada estágio, sendo similar ao analisado em [15].

\section{Desempenho e Complexidade de Sistemas DS-UWB}

Analisa-se nesta seção a relação de compromisso desempenho versus complexidade do sistema DS-UWB, com o objetivo de se obter uma topologia capaz de oferecer desempenho razoável sem fazer uso de grande complexidade computacional. Os custos computacionais - e conseqüentemente os de implementação - para as distintas configurações do sistema DS-UWB (SRake, PIC, múltiplas antenas) são analisadas tendo em vista o estabelecimento de uma figura de mérito capaz de quantificar o compromisso desempenhocomplexidade para sistemas DS-UWB.

A Tabela I apresenta os principais parâmetros utilizados nas simulações computacionais Monte-Carlo (MCS) do sistema DS-UWB em canal multipercurso denso.

TABELA I

PARÂMETROS UTILIZADOS NOS SISTEMAS DS-UWB.

\begin{tabular}{|l|c|}
\hline Parâmetro & Valor \\
\hline \hline Tipo de pulso & Gaussiano, 2a. derivada \\
\hline Fator de formatação de pulso, $\tau_{p}$ & $287,7 \mathrm{ps}$ \\
\hline Período de quadro $($ frame $), T_{f}$ & $279 \mathrm{~ns}$ \\
\hline Duração de bit, $T_{b}$ & $27,9 \mathrm{~ns}$ \\
\hline Período de chip, $T_{c}$ & $0,9 \mathrm{~ns}$ \\
\hline Período de pulso & $0,7 \mathrm{~ns}$ \\
\hline Número de usuários, $U$ & 11 \\
\hline Seqüência de espalhamento & Gold \\
\hline Número de chips por bit, $N_{c}$ & 31 \\
\hline Canal S-V Modificado & CM-3 (NLOS 4-10m) \\
\hline $\begin{array}{l}\text { Porcentagem da energia total } \\
\text { do canal utilizada }\end{array}$ & $85 \%$ \\
\hline Número de ramos no SRake, $L_{f}$ & 5 ou 10 \\
\hline Número de estágios PIC, $S$ & 1 ou 2 \\
\hline Número de antenas no Rx, $M$ & 1,2 ou 3 \\
\hline Número de erros/ponto $(\mathrm{MCS})$ & $>20$ \\
\hline
\end{tabular}

\section{A. Desempenho UWB com Cancelamento de Interferência Paralelo, Diversidade de Percurso e Espacial}

Os resultados de simulação MCS obtidos nas Figuras 1 e 2 permitem analisar a influência conjunta das diversidades espacial e temporal, e da detecção multiusuário (detecção PIC) sobre o desempenho de sistemas DS-UWB em ambiente multipercurso denso. Nestas figuras, considerou-se o efeito do sombreamento (perfil não normalizado), canal com $85 \%$ da energia total e uma janela de processamento no receptor correspondente a 10 símbolos .

A utilização das estratégias PIC e múltiplas antenas resulta em melhoria significativa de desempenho em relação ao detector convencional (SRake). Da Figura 1, nota-se melhoria substancial de desempenho combinando-se estágios PIC ( $S=$ 1 ou 2) e mais de uma antena no receptor $(M=2$ ou 3 antenas receptoras). Melhoria adicional de desempenho é obtida quando o número de ramos do receptor SRake é dobrado, Figura 2. Comparando-se os resultados de desempenho das Figuras 1 e 2 , para alta região de $E_{b} / N_{0}$, verifica-se que o desempenho do SRake MRC (sem PIC) com 3 antenas receptoras da Figura 1 é equivalente ao desempenho do SRake MRC com 1 estágio PIC e uma antena receptora na Figura 2. Para $M=2$ antenas com PIC, há uma melhoria de desempenho de, aproximadamente, uma década com uso de $L_{f}=10$ ramos ao invés de 5 ramos.

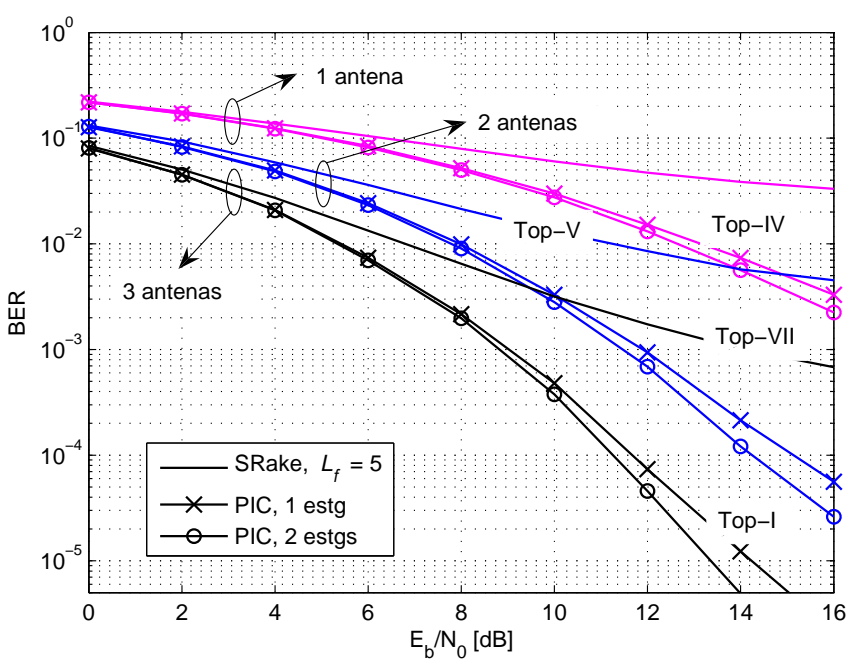

Fig. 1. BER para sistema DS-UWB para canal SV modificado CM-3, SRake com 5 ramos, 1 e 2 estágios PIC combinado com recepção através de 1 a 3 antenas; Load $=\frac{U}{N_{c}}=\frac{11}{31} \approx 0,33$.

Note que a melhoria de desempenho alcançado com o $2^{\circ}$ estágio PIC na Figura 1 só é significativa na região de alto $E_{b} / N_{0}$ (acima de $14 \mathrm{~dB}$ ).

\section{B. Aspectos de Complexidade de Implementação}

A análise da complexidade computacional de um determinado algoritmo pode ser realizada através da ordem de grandeza de sua complexidade, representada pela notação $\mathcal{O}$. Por exemplo, a complexidade do sistema DS-UWB necessária ao processamento, no domínio do tempo, de um usuário é $\mathcal{O}_{\text {Rake }}\left(L_{f} P N_{c}\right)$, onde $P$ é o número de símbolos processados [23].

Por simplicidade, as operações de multiplicação e divisão foram consideradas como operações principais e as operações de adição e subtração foram desconsideradas por apresentarem tempo computacional ínfimo em comparação com as operações de multiplicação e divisão. 


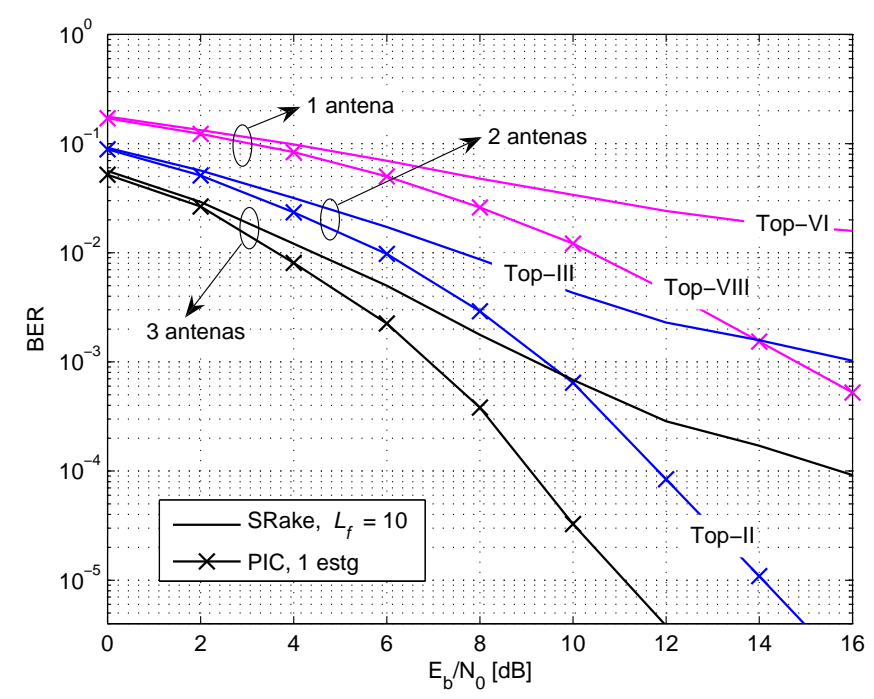

Fig. 2. BER para sistema DS-UWB para canal SV modificado CM-3, SRake com 10 ramos e 1 estágio PIC combinado com recepção através de 1 a 3 antenas. $\operatorname{Load} \approx 0,33$

Pode-se expressar a complexidade computacional para o receptor processar o sinal DS-UWB e estimar um símbolo $(P=1)$, em termos de instruções executadas, conforme descrito na Tabela II. Esta complexidade refere-se ao link unidade portátil-central ${ }^{3}$. Observe que para o caso do link unidade central-portátil ${ }^{4}$, a utilização do detector MuD PIC é ainda muito menos justificável devido ao incremento de processamento na unidade portátil.

TABELA II

COMPLEXIDADE DOS RECEPTORES DS-UWB ANALISADOS EM TERMOS DO NÚMERO DE OPERAÇÕES CONSIDERANDO $M$ ANTENAS.

\begin{tabular}{l|c}
\hline Topologia & Número de operações \\
\hline \hline Rake & $U \cdot L_{f} \cdot\left(N_{c}+1\right) \cdot M$ \\
\hline PIC & $U \cdot L_{f} \cdot\left(3 N_{c}+1\right) \cdot S \cdot M$ \\
\hline Rake + PIC & $U \cdot L_{f} \cdot M\left[(S+1)+\left(N_{c}(3 S+1)\right)\right]$ \\
\hline
\end{tabular}

Observe que a complexidade computacional do sistema DSUWB é proporcional ao número de percursos recuperados. Com isso, a complexidade computacional da etapa de desespalhamento torna-se elevada em ambientes multipercurso. Por esta razão, deve-se procurar outras formas de melhoria de desempenho sem a necessidade de processamento de um grande número de percursos para recuperar o sinal.

\section{Compromisso Desempenho-Complexidade}

A partir dos resultados de desempenho, considerando canal SV modificado CM-3, analisou-se a complexidade computacional resultante para cada topologia de recepção: SRake, SRake com PIC, e também com a combinação de até 3 antenas.

A Figura 3 mostra a complexidade computacional levandose em consideração o número de estágios PIC utilizados (zero estágio corresponde ao receptor na ausência de cancelador de interferência, apenas o SRake). Esta figura indicou que 2 antenas seguidas por SRake com 5 ramos resulta na mesma

\footnotetext{
${ }^{3}$ Análogo ao link reverso em telefonia celular.

${ }^{4}$ Equivalente ao link direto em telefonia celular.
}

complexidade da configuração do receptor com 1 antena e SRake com 10 ramos. Neste caso, em termos de complexidade, acrescentar 5 ramos é equivalente a acrescentar 1 antena. Porém, resultados de desempenho para CM-3 mostram que 2 antenas com 5 ramos apresenta melhor desempenho do que considerar receptor com 1 antena e SRake de 10 ramos; esta melhoria em termos de taxa de erro de bit (BER) é da ordem de uma década quando utiliza-se um estágio PIC.

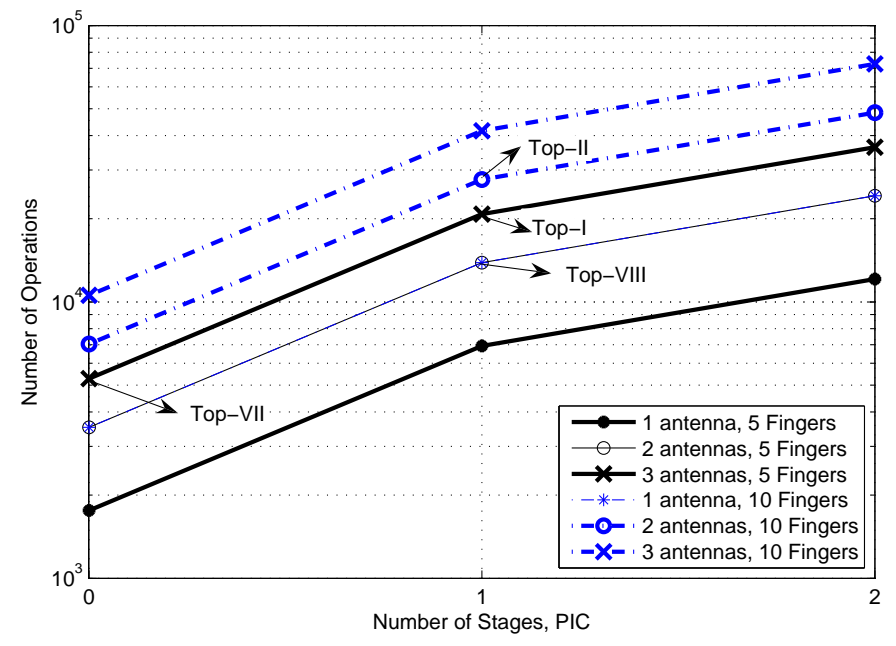

Fig. 3. Complexidade computacional para sistema DS-UWB considerando 1 a 3 antenas, 0 a 2 estágios PIC e SRake com 5 ou 10 ramos.

Das figuras de desempenho analisadas, identificam-se dois pares de topologias com desempenho semelhantes (Tabela III).

TABELA III

TOPOLOGIAS COM DESEMPENHOS SEMELHANTES.

\begin{tabular}{cccccc}
\hline Top & \# ant., $M$ & \# ramos, $L_{f}$ & \# $S$ PIC & Complex. & Fig. \\
\hline \hline I & 3 & 5 & 1 & menor & 1 \\
II & 2 & 10 & 1 & maior & 2 \\
\hline VII & 3 & 5 & 0 & menor & 6 \\
VIII & 1 & 10 & 1 & maior & 6 \\
\hline
\end{tabular}

Porém, plotando-se as curvas de complexidade (em termos do número de operações computacionais relevantes) em função do número de estágios PICs, parametrizadas pelo número de antenas e ramos, Figura 3, verifica-se facilmente que a Top-I apresenta complexidade menor do que a Top-II. As topologias Top-VII e Top-VIII possuem desempenhos semelhantes para alta região de $E_{b} / N_{0}$, sendo que a Top-VII possui menor complexidade em termos de operações executadas. Nas regiões de média e baixa $E_{b} / N_{0}$, a Top-VII possui melhor desempenho.

Em seguida, as situações com complexidades computacionais semelhantes, porém com desempenhos distintos são analisadas. A Figura 4 mostra a complexidade computacional em função do carregamento do sistema (Load), considerando dois casos: (a) uma única antena, SRake com 5 e 10 ramos seguido do detector MUD-PIC; (b) ausência de detecção multiusuário, porém considerando 1, 2 e 3 antenas e detecção convencional SRake com 5 e 10 ramos. Identificam-se dois pares de topologias receptoras com complexidades computacionais semelhantes (Tabela IV). 
TABELA IV

TOPOLOGIA COM COMPLEXIDADES COMPUTACIONAIS SEMELHANTES.

\begin{tabular}{cccccc}
\hline Top & \# ant., $M$ & \# ramos, $L_{f}$ & \# $S$ PIC & Perform. & Fig. \\
\hline \hline III & 2 & 10 & 0 & melhor & $4 . \mathrm{b}$ \\
IV & 1 & 5 & 1 & pior & $4 . \mathrm{a}$ \\
\hline V & 2 & 5 & 0 & melhor & $4 . \mathrm{b}$ \\
VI & 1 & 10 & 0 & pior & $4 . \mathrm{b}$ \\
\hline
\end{tabular}

Da figura 4, verifica-se que a Top-III resulta na mesma complexidade da Top-IV. No entanto, a Top-III apresenta melhor desempenho do que a Top-IV. Analogamente, a Top-V resulta na mesma complexidade de Top-VI, porém a Top-V apresenta desempenho superior à Top-VI.

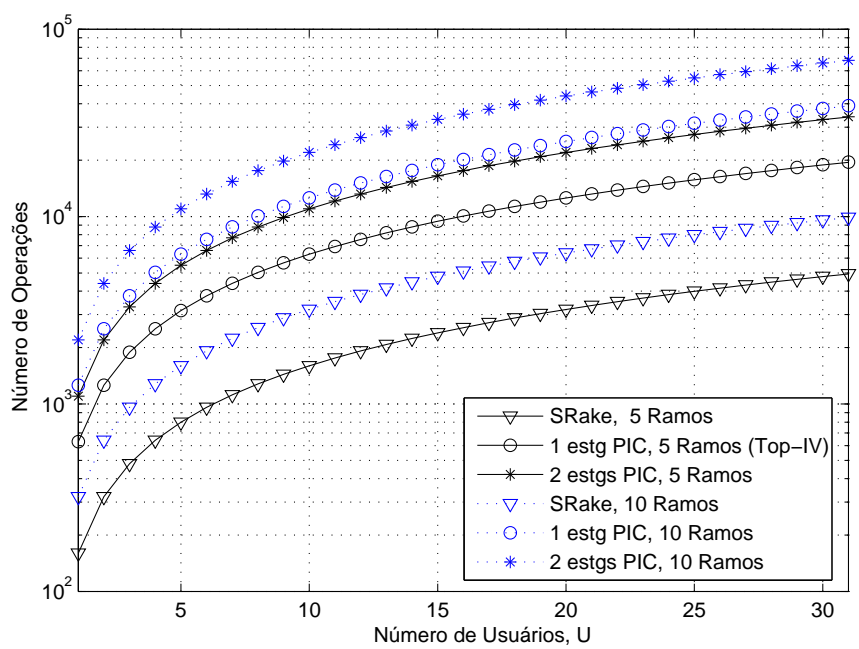

(a)

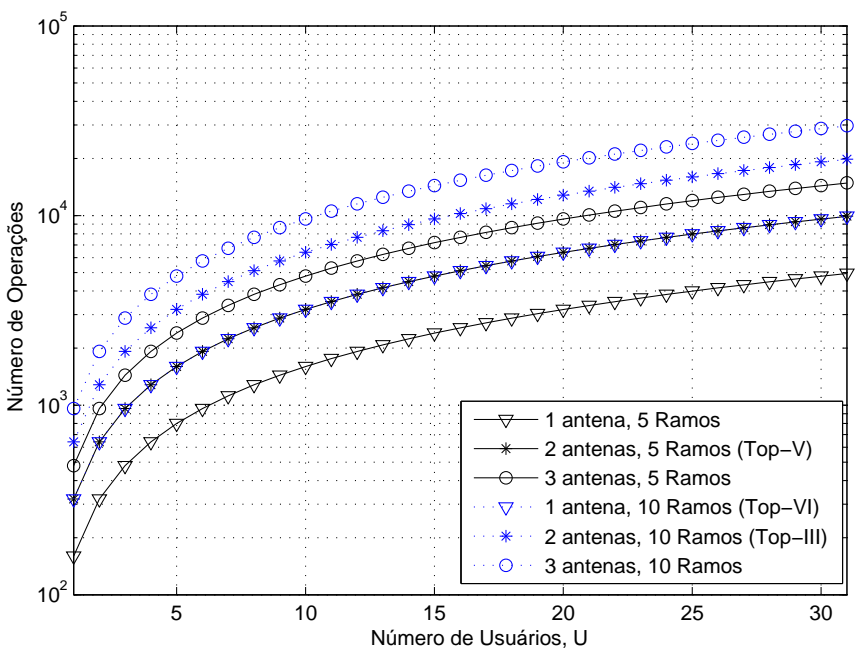

(b)

Fig. 4. Complexidade computacional para sistema DS-UWB: (a) com apenas uma antena; SRake com 5 ou 10 ramos seguido de 1 estágio PIC; (b) 1 a 3 antenas e SRake com 5 ou 10 ramos.

Rearranjando os resultados anteriores, evidencia-se na Figura 5 o incremento da complexidade computacional do Receptor DS-UWB em função do carregamento do sistema para duas situações:

a) quando há um aumento no número de ramos no receptor DS-UWB SRake, considerando uma única antena; b) duas topologias distintas com desempenhos semelhantes.

A Figura 5.a mostra o acréscimo de complexidade quando o número de ramos é incrementado de 5 para 10 . Nota-se um expressivo aumento no custo computacional ao se acrescentar um estágio PIC em comparação à duplicação do número de ramos do SRake. Na Figura 5.b são consideradas duas situações de desempenhos DS-UWB semelhantes em canais SV CM-3 para alta região de $E_{b} / N_{0}$, porém com complexidades computacionais distintas. Com isso, fica evidenciado mais uma vez que a exploração da diversidade espacial em receptores DS-UWB resulta em melhor compromisso desempenho-complexidade em relação ao uso da detecção multiusuário.
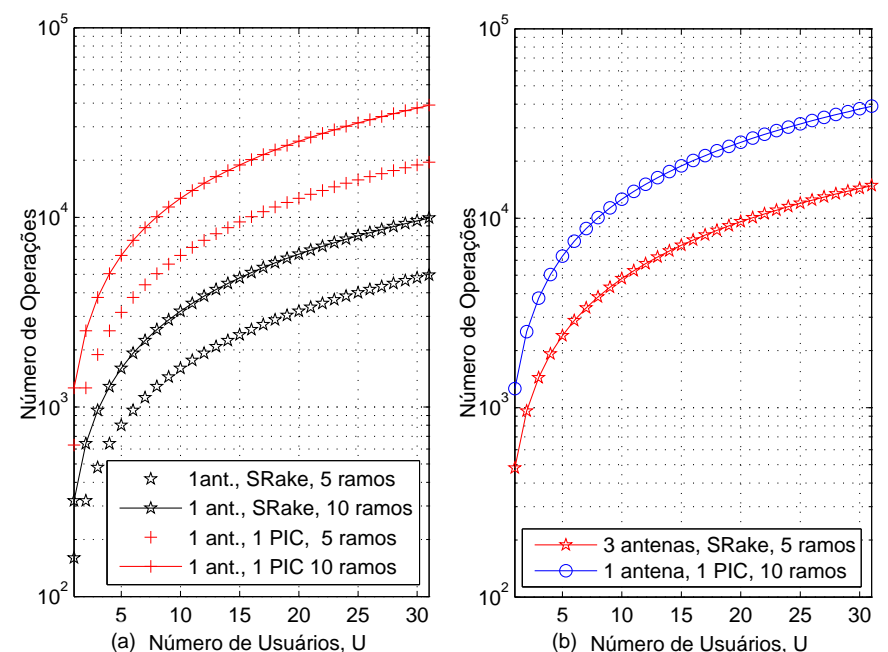

Fig. 5. Complexidade do Rx DS-UWB: a) SRake e com PIC-MuD, considerando 5 e 10 ramos; b) Desempenhos semelhantes em canal SV CM-3, para alta região de $E_{b} / N_{0}$.

\section{CONCLUSÃO}

O sistema DS-UWB foi analisado através de simulação Monte-Carlo com o intuito de se estabelecer uma figura de mérito desempenho-complexidade, assim como demonstrar a influência das diversidades temporal, espacial e também de detecção multiusuário no desempenho do sistema em ambiente multipercurso denso. Esta melhoria de desempenho, obtida através do uso de múltiplas antenas receptoras e/ou de cancelador de interferência, pôde ser comparada e quantificada para os casos considerados. Obteve-se melhoria marginal com a utilização do cancelador PIC de 2 estágios em relação ao PIC com 1 estágio. Esta pequena diferença de desempenho é ainda menor à medida que o número de ramos do SRake aumenta, não justificando o uso do cancelador PIC com mais de 1 estágio.

Adicionalmente, resultados de desempenho MCS mostram que a utilização de 2 ou 3 antenas oferece ganho de desempenho proporcional em relação ao uso de uma única antena receptora. Combinando-se distintos arranjo de antenas receptoras ( $M \leq 3$ ), ramos do SRake , e/ou estágios PIC, foram obtidas topologias receptoras DS-UWB com desempenhos semelhantes, porém complexidades diferentes. Assim, da análise de complexidade computacional, conclui-se que é melhor aumentar o número de antenas ou o número de ramos 
do que utilizar um ou mais estágios PIC para se atingir um determinado desempenho nas configurações analisadas.

\section{REFERÊNCIAS}

[1] M. Z. Win, R. A. Scholtz; "Impulse radio: how it works," IEEE Communications Letters, vol. 2, no. 2, Feb. 1998, pp. 36-38

[2] J. R. Foerster; "Channel Modeling Sub-committee Report Final," IEEE 802.15-02/490; Disponível: http://ieee802.org/15/.

[3] A. F. Molisch, J. R. Foerster, M. Pendergrass; "Channel models for ultrawideband personal area networks," IEEE Wireless Communications, vol. 10, no. 6, Dec. 2003, pp. 14-21.

[4] M. Z. Win and R. A. Scholtz; "Ultra-wide bandwith timehopping spread-spectrum impulse radio for wireless multipleaccess communications," IEEE Trans. Commun., vol. 48, no. 4, Apr. 2000, pp. 679-689.

[5] J. R. Foerster; "The performance of a direct-sequence spread ultrawideband system in the presence of multipath, narrowband interference, and multiuser interference," IEEE Conference on Ultra Wideband Systems and Technologies, May 2002, pp. 8791.

[6] J. D. Choi, W. E. Stark; "Performance of Ultra-Wideband communications with suboptimal receivers in multipath channels", IEEE J. Sel. Areas Commun., vol. 20, no. 9, Dec. 2002, pp. $1754-1766$.

[7] J. R. Foerster; "The Effects of Multipath Interference on the Performance of UWB Systems in an Indoor Wireless Channel," IEEE 53rd Vehicular Technology Conference, vol. 2, May 2001, pp. 1176-1180.

[8] D. Cassioli, M. Z. Win, F. Vatalaro, A. F. Molisch; "Performance of low-complexity RAKE reception in a realistic UWB channel," IEEE International Conference on Communications, Jan. 2002, pp. 763-767.

[9] M. Z. Win and R. A. Scholtz; "On the energy capture of ultrawide bandwidth signals in dense multipath environments," IEEE Commun. Lett., vol. 2, no. 9, Sep. 1998, pp. 245-247.

[10] J.D. Choi and W. E. Stark; "Performance analysis of Rake receivers for ultra-wideband communications with PPM and OOK in multipath channels," in Proc. IEEE ICC, Apr. 2002, vol. 3, pp. 1969-1973.

[11] X. Shen, W. Zhuang, H. Jiang, J. Cai; "Medium access control in ultra-wideband wireless networks," IEEE Transactions on Vehicular Technology, vol. 54, no. 5, Sep. 2005, pp. 1663-1677.

[12] R. C. Qiu, H. Liu, X. Shen; "Ultra-wideband for multiple access communications," IEEE Communications Magazine, vol. 43, no. 2, Feb. 2005, pp. 80-87.

[13] A. Saleh and R. Valenzuela; "A statistical model for indoor multipath propagation", IEEE J. Sel. Areas Commun., vol. 5, no. 2, Feb. 1987, pp. 128-137.

[14] A. Papoulis, "Probability, Random Variables and Stochastic Processes," Electrical Engineering. Communications and Signal Processing. McGraw-Hill, $3^{\text {rd }}$ edition, pp. 290, 1991.

[15] L. Heringer, "Desempenho e Complexidade de Sistemas DSUWB em Canais Multipercursos Densos. Dissertação de Mestrado, 151 pgs, Universidade Estadual de Londrina - UEL, 2007.

[16] B. A. Angélico, L. C. Heringer, T.Abrão, P. J. E. Jeszensky; "Ultra-wideband Performance in a Dense Multipath Environment with Time and Spatial Diversity", IST'07 - The 16th IST Mobile and Wireless Communications Summit, Budapeste, Hungria, 2007.

[17] A. F. Molisch, J. R. Foerster, M. Pendergrass; "The Evolution of Wireless LANs and PANs-Channel Models for Ultrawideband Personal Area Networks," IEEE Transactions on Wireless Communications, vol. 10, no. 6, December 2003, pp. 14-21.

[18] M. A. Peyrot-Solis, G. M. Galvan-Tejada, H. Jardon-Aguilar; "State of the art in ultra-wideband antennas," 2nd International Conference on Electrical and Electronics Engineering, Mexico, September 2005, pp. 101-105.
[19] R. Lupas, S. Verdú; "Linear Multiuser Detectors for Synchronous CDMA Channels," IEEE Transactions on Information Theory, vol. 35, no. 1, January 1989, pp. 123-136.

[20] S. Moshavi; "Multi-User Detection for DS-CDMA Communicatios," IEEE Communications Magazine, 1A, October 1996, pp. 124-136.

[21] Z. Zvonar, A. Duel-Hallen; "Multiuser detection for CDMA systems," IEEE Personal Communications, vol. 2, no. 2, April 1995, pp. 46-58.

[22] T. Abrão; "Canceladores de Interferência Multiusuário Aplicados a Sistemas DS/CDMA de Múltipla Taxa," Tese de Doutorado - Escola Politécnica da Universidade de São Paulo - EPUSP, 2001.

[23] H. Sato, T. Ohtsuki, "Frequency domain channel estimation and equalisation for direct sequence ultra wideband (DS-UWB) system," IEE Proceedings Communications, vol. 153, no. 1, Feb. 2006, pp. 93-98. 